\title{
MELAS: A Multigenerational Impact of the MTTL1 A3243G MELAS Mutation
}

\author{
M. Prasad, B. Narayan, A.N. Prasad, C.A. Rupar, S. Levin, J. Kronick, \\ D. Ramsay, K.Y. Tay, C. Prasad
}

\begin{abstract}
Background: The maternally inherited MTTL1 A3243G mutation in the mitochondrial genome causes MELAS (Mitochondrial Encephalopathy Lactic Acidosis with Stroke-like Episodes), a condition that is multisystemic but affects primarily the nervous system. Significant intra-familial variation in phenotype and severity of disease is well recognized. Methods: Retrospective and ongoing study of an extended family carrying the MTTL1 A3243G mutation with multiple symptomatic individuals. Tissue heteroplasmy is reviewed based on the clinical presentations, imaging studies, laboratory findings in affected individuals and pathological material obtained at autopsy in two of the family members. Results: There were seven affected individuals out of thirteen members in this three generation family who each carried the MTTL1 A3243G mutation. The clinical presentations were varied with symptoms ranging from hearing loss, migraines, dementia, seizures, diabetes, visual manifestations, and stroke like episodes. Three of the family members are deceased from MELAS or to complications related to MELAS. Conclusions: The results of the clinical, pathological and radiological findings in this family provide strong support to the current concepts of maternal inheritance, tissue heteroplasmy and molecular pathogenesis in MELAS. Neurologists (both adult and paediatric) are the most likely to encounter patients with MELAS in their practice. Genetic counselling is complex in view of maternal inheritance and heteroplasmy. Newer therapeutic options such as arginine are being used for acute and preventative management of stroke like episodes.
\end{abstract}

RÉSUMÉ: MELAS : Impact multigénérationnel de la mutation MTTL1 A3243G. Contexte : La mutation MTTL1 A3243G, transmise par la mère dans le génome mitochondrial, cause MELAS (acidose lactique liée à une encéphalopathie mitochondriale, avec des épisodes ressemblant à des accidents vasculaires cérébraux), une maladie multisystémique qui atteint principalement le système nerveux. Il existe des variations intrafamiliales du phénotype et de la sévérité de la maladie. Méthode : Nous rapportons une étude rétrospective et prospective d'une famille étendue porteuse de la mutation MTTL1 A3243G et dont plusieurs membres présentent des symptômes de la maladie. Nous revoyons l'hétéroplasmie tissulaire en lien avec la présentation clinique, les études d'imagerie, les résultats de laboratoire chez les individus atteints et le matériel anatomopathologique obtenu à l'autopsie chez deux des membres de la famille. Résultats : Sept individus répartis sur 3 générations parmi les 13 membres de la famille étaient atteints et chacun d'eux était porteur de la mutation MTTL1 A3243G. Le tableau clinique variait d'un individu à l'autre, dont une perte auditive, des migraines, de la démence, des crises convulsives, du diabète, des manifestations visuelles et des épisodes ressemblant à un accident vasculaire cérébral. Trois des membres de la famille sont décédés de MELAS ou de complications reliées au MELAS. Conclusions : Les constatations cliniques, anatomopathologiques et radiologiques faites dans cette famille appuient les concepts actuels sur le MELAS, soit une hérédité maternelle, une hétéroplasmie tissulaire et une pathogenèse moléculaire. Les neurologues, tant adultes que pédiatriques, sont ceux qui sont les plus susceptibles de rencontrer des individus atteints de MELAS parmi leurs patients. Le conseil génétique est complexe, compte tenu de l'hérédité maternelle et de l'hétéroplasmie. De nouvelles options thérapeutiques telle l'arginine sont utilisées pour le traitement aigu et préventif des épisodes ressemblant à un AVC.

Can J Neurol Sci. 2014; 41: 210-219

Mitochondrial Encephalopathy, lactic acidosis, and stroke like episodes (MELAS), first described in 1984, is one of the most common mitochondrial disorders with a prevalence of 0.06 $\%$ in the general population ${ }^{1}$. Mitochondrial disorders affect multiple organ systems and tissues which consequently causes a number of "overlaps" or multisystem presentations resulting in varied phenotypes. The triad of stroke-like episodes, seizures and lactic acidosis clinically distinguishes MELAS from other mitochondrial disorders ${ }^{1}$. However, as seen in this family, not all individuals with the MTTL1 A3243G mutation present clinically with MELAS.

This disorder is most frequently associated with an A to $G$ transition at position 3243 in the mitochondrial genome ${ }^{2}$. This mutation occurs in the tRNA ${ }^{\text {leucine }}$ gene, which is the site of many disease associated mutations ${ }^{3}$. The mutation alters the structure, stability, methylation, amino-acylation and codon recognition properties of mitochondrial $\mathrm{DNA}^{3}$. As a result, the pairing of the mRNA codon with the mutant tRNA anticodon is hindered,

From the Departments of Paediatrics (ANP, CAR, SL, CP), Pathology and Laboratory Medicine (CAR, DR), Medical Imaging (KYT), Biochemistry (CAR), Children's Health Research Institute (CAR, CP), 1Western University and London Health

Sciences Centre (MP, BN), London; Department of Paediatrics (JK), Hospital for Sick Children, Toronto, Ontario, Canada.

Received April 23, 2013. Final Revisions Submitted OCtober 10, 2013. Correspondence to: Chitra Prasad, Children's Hospital, London Health Sciences Centre, 800 Commissioners Road East, London, Ontario, N6C 2V5, Canada. Email: Chitra.Prasad@lhsc.on.ca. 
leading to inaccurate tRNA processing, impaired translation, lowered rates of protein synthesis and enzyme activity ${ }^{3}$. A significant negative correlation between the percentage of MTTL1 A3243G mutation and the specific activity of mitochondrial respiratory chain complex I, the respiratory chain complex with the highest number of mtDNA-encoded subunits, has been reported ${ }^{3}$.

The phenotypic variability in MELAS includes: sensorineural hearing loss, migraine headaches, dementia, depression, learning difficulties, diabetes, short stature associated with poor growth, and cardiomyopathy ${ }^{1}$. In the present multigenerational family study we describe the evidence supporting heteroplasmy at a molecular level contributing to different clinical phenotypes in the affected family members.

\section{Materials AND Methods}

Ethics approval was obtained from Western University Health Sciences Ethics Review Board. Clinical and laboratory records such as magnetic resonance imaging (MRI) reports,

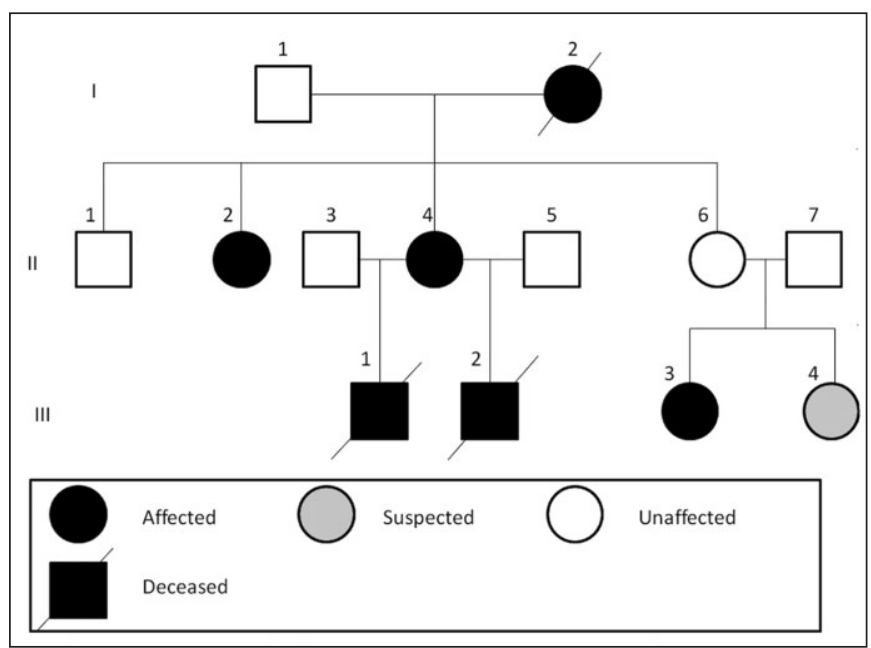

Figure 1: Pedigree of the family studied.

\section{Table 1: Symptom Chart and mtDNA analysis}

\begin{tabular}{|c|c|c|c|c|c|c|c|c|}
\hline & III-1 & III-2 & II-4 & $\mathbf{I}-2$ & III-3 & III-4 & II-2 & II-6 \\
\hline Strokes & + & + & + & & & & + & \\
\hline Seizures & + & + & + & & & & & \\
\hline Dementia & & & & + & & & & \\
\hline Hearing Loss & + & + & + & + & & & + & \\
\hline Learning Difficulties & + & + & + & & & & & \\
\hline Migraines & + & + & + & & + & & & \\
\hline Weakness & + & + & & & & & & \\
\hline Exercise Intolerance & + & + & & & & & & \\
\hline Psychiatric Problems & + & + & + & + & + & + & + & \\
\hline Diabetes & & & + & & & & + & \\
\hline Blindness & + & & & & & & & \\
\hline Premature Aging & & & & + & & & + & \\
\hline \multicolumn{9}{|l|}{ DNA Source } \\
\hline Muscle & $76(18)$ & & & $39-59(65)$ & & & & \\
\hline Cultured skin fibroblasts & $54(18)$ & & & $58(65)$ & & & & \\
\hline Urine epithelial cells & & & $66(49)$ & & & $28(14)$ & & \\
\hline Pancreas & & & & $31(65)$ & & & & \\
\hline Brain & & & & $67(65)$ & & & & \\
\hline Blood lymphocytes & $49(11)$ & $50(8)$ & $16(31)$ & $4(65)$ & $5(4)$ & $\begin{array}{c}\text { Not } \\
\text { detected } \\
(16 \\
\text { months })\end{array}$ & $\begin{array}{c}15 \\
(32)\end{array}$ & $\begin{array}{c}5 \\
(30)\end{array}$ \\
\hline Kidney & & & & $35(65)$ & & & & \\
\hline Liver & $84(18)$ & & & $28(65)$ & & & & \\
\hline Myocardium & & & & $73(65)$ & & & & \\
\hline Cerebellum & $19-27(18)$ & & & $53(65)$ & & & & \\
\hline Thyroid & & & & $78(65)$ & & & & \\
\hline Lung & & & & $81(65)$ & & & & \\
\hline Spleen & & & & $3(65)$ & & & & \\
\hline Brainstem & $78(18)$ & & & & & & & \\
\hline Cerebral cortex & $51-60(18)$ & & & & & & & \\
\hline
\end{tabular}

+ sign indicates that the patient has that particular symptom; () indicate the age at which testing was done 
autopsies, and electrical encephalophagram (EEG) findings were reviewed and data were abstracted on affected individuals in this family. However, muscle biopsies during life were not performed for any of the individuals, so only III-1 and I-2's muscle biopsy (post mortem) were analyzed.

\section{Clinical Data}

The family pedigree is outlined in Figure 1. The summary of descriptions of clinical symptoms, laboratory findings and DNA analysis is provided in Tables 1 and 2, respectively.

\section{III-1 (Proband) (Figure 1)}

The proband was born at 35 weeks gestation to a 20 -year-old mother of Scottish Irish background with a birth weight of 2.438 $\mathrm{kg}$. His symptom complex that developed over several years included; "vascular" migraine headaches, stroke like episodes involving the right occipital lobe, failure to thrive, focal, and secondarily generalized seizures, weakness, intermittent diplopia, vomiting, failure to thrive, developmental delay, attention deficit disorder, depression and acting out behaviours.

At age eleven, the proband presented with a focal seizure following minor head trauma involving left-hemibody clonic activity and conjugate gaze deviation to the left followed by a
Todd's left hemiparesis. In addition, he complained of blurred vision and weakness. The ictus was followed by persistent vomiting for an hour and ataxia. A month later, he again presented with left hemibody focal seizures in association with a tonsillar infection, a left visual field defect and headache. His examination at age 15 years showed mild ptosis, intact extraocular movements, absence of nystagmus, and a partial left inferior quadrantanopsia on visual field testing. Intermittent diplopia was attributed to his antiepileptic medication Dilantin. His motor examination was significant for the presence of normal power, variable tone, polymyoclonus, and generalized hyporeflexia. The EEG revealed a moderate diffuse slowing of background rhythms on which was superimposed focal slowing in the right posterior occipito- parietal and temporal regions, the findings were compatible with the presence of MRI findings consistent with right occipital infarction. Based on a clinical triad of recurrent seizures, mild lactic acidosis (2.5-2.8) (reference range $0.5-2.2 \mathrm{mmol} / \mathrm{L}$ ) and stroke like episodes, MELAS was suspected and later confirmed by DNA analysis (Table 1). A muscle biopsy was not done, as a molecular diagnostic test was available on site. His medications included; valproic acid (prior to diagnosis of mitochondrial disorder), clobazam (seizures), vitamin C, succinic acid, and Coenzyme

Table 2: Laboratory findings

\begin{tabular}{|c|c|c|c|c|c|}
\hline Patient & Lactate & MRI \& MRS & $\begin{array}{l}\text { Muscle Biopsy } \\
\text { (Post-mortem) }\end{array}$ & EEG & $\begin{array}{l}\text { Respiratory Chain Studies } \\
\text { (Performed with autopsy tissue) }\end{array}$ \\
\hline III-1 & $2.0-5.8$ & $\begin{array}{l}\text { Large region of } \\
\text { signal abnormality } \\
\text { involving cortical } \\
\text { regions of the left } \\
\text { parieto-occipital lobe. } \\
\text { Region of increased } \\
\text { signal on diffusion in } \\
\text { left cerebellar } \\
\text { hemisphere }\end{array}$ & $\begin{array}{l}\text { No "true" ragged } \\
\text { red fibres. } \\
\text { Necrotising } \\
\text { "myopathy" } \\
\text { pattern. }\end{array}$ & $\begin{array}{l}\text { Focal delta activity in } \\
\text { the right posterior } \\
\text { occipital-parietal and } \\
\text { temporal region } \\
\text { superimposed on } \\
\text { generalised slowing } \\
\text { moderate diffuse } \\
\text { disturbance of } \\
\text { cerebral function }\end{array}$ & $\begin{array}{l}\text { Enhanced succinate } \\
\text { dehydrogenase (SDH) and } \\
\text { NADH activity associated } \\
\text { with attenuated cytochrome C } \\
\text { oxidase activity (COX). }\end{array}$ \\
\hline III-2 & $\begin{array}{l}4.5-9.8 \\
\text { (Elevated } \\
\text { lactate) }\end{array}$ & $\begin{array}{l}\text { Area of increased T2 } \\
\text { signal in left occipital } \\
\text { lobe. MRS showed } \\
\text { inverted lactate peak } \\
\text { in the right occipital } \\
\text { lobe }\end{array}$ & N/A & $\begin{array}{l}\text { Abundant generalised } \\
\text { epileptic discharges }\end{array}$ & \\
\hline II-4 & 2 & $\begin{array}{l}\text { Extensive white } \\
\text { matter changes } \\
\text { ventricular } \\
\text { enlargement right } \\
\text { sided cerebral } \\
\text { atrophy }\end{array}$ & $\mathrm{N} / \mathrm{A}$ & $\begin{array}{l}\text { Mild to moderate } \\
\text { diffuse slowing }\end{array}$ & N/A \\
\hline I-2 & N/A & N/A & $\begin{array}{l}\text { Mild } \\
\text { myopathy } \\
\text { Sparse } \\
\text { ragged red } \\
\text { fibres }\end{array}$ & N/A & N/A \\
\hline II-2 & 2.5 & N/A & N/A & N/A & N/A \\
\hline III-3 & $0.5-2.2$ & $\begin{array}{l}\text { No areas of signal } \\
\text { abnormality }\end{array}$ & N/A & N/A & $\mathrm{N} / \mathrm{A}$ \\
\hline III-4 & 0.5 to 2.2 & $\begin{array}{l}\text { No areas of signal } \\
\text { abnormality. } \\
\text { No lactate is seen } \\
\text { in MRS. }\end{array}$ & $\mathrm{N} / \mathrm{A}$ & N/A & N/A \\
\hline
\end{tabular}


Q10 (supplements for mitochondrial disorder). At age sixteen he experienced a significant deterioration, developed cortical blindness, and lost the ability to speak and feed himself. Further decline in cognition followed and the proband died at the age of 18 from aspiration pneumonia. An autopsy was performed, the details of which are included in the pathology section.

\section{III-2}

The proband's half-brother was evaluated at age eight due to his family history. He was delivered at term by Caesarean section, which was carried out on account of significant fetal decelerations. Birth weight was 2863 gms (50th percentile). He was diagnosed with MELAS at 11 years when he developed a progressive neurological syndrome that included; migraines, stroke-like episodes, and encephalopathy characterized by confusion, double vision and visual hallucinations, recurrent focal and generalized seizures, polymyoclonus. The headaches were bifrontal, associated with a pounding character, scintillating scotomata, nausea and vomiting. He was also found to carry the MTTL1 A3243G mutation in his blood leukocytes (Table 1). At age 17 years, he underwent visual field testing with Goldmann perimetry which disclosed a right superior homonymous quadrantanopia. Although he complained of double vision, his neurological examination did not show any ptosis or paresis of extraocular muscle. The remainder of his examination was significant for normal power, variable tone, normal cerebellar and gait testing. EEG findings showed temporal changes. Initially, generalized spike and wave abnormalities and a self limited photoparoxymsal response were reported in association with generalized tonic clonic seizures. In later years focal slowing particularly noticeable over posterior quadrants as well as more moderate diffuse slowing (theta and delta frequencies) of background rhythms were noted. Serial MRI scans were also performed during multiple admissions. Nerve conduction studies were performed serially, nerves examined included the median and ulnar nerves in the upper extremity, common peroneal, deep peroneal, and tibial nerves in the lower extremity. While nerve conduction velocities were normal, a reduction in the amplitude of the compound muscle action potential (CMAP) from the extensor digitorum brevis muscle was the sole abnormality identified. Sensory nerve action potentials were normal even on repeated examinations in the sural and superficial peroneal nerves. Over the next five years, he experienced progressive cognitive decline, lost the ability to ambulate independently and was eventually confined to a wheelchair due to physical disability, headaches, recurrent seizures and stroke like episodes with significant visual hallucinations (Table 1). He also had significant gut motility issues, particularly constipation requiring intensive bowel management. At this stage he was noted to have myoclonic jerks, a tremor of his hands which was attributable to polymyoclonus. He was treated with multiple medications; dichloroacetate (was discontinued due to symptoms of peripheral neuropathy and no major benefit reported in literature ${ }^{1}$ ), vitamin $\mathrm{K}$, carnitine, Larginine, riboflavin, thiamine, vitamin C, pizotifen (migraine), topiramate (seizures), risperidone (behavioural outbursts) oxybutynin (bladder dysfunction), Coenzyme Q10, vitamin E, and periods of total parenteral nutritional supplementation. He died at age 18 from intractable seizures, encephalopathy and respiratory failure. There was no autopsy performed and a muscle biopsy during life was not performed.

\section{II-4}

The proband's mother had a history of mild intellectual disability, diabetes, hypertension, bilateral sensorineural hearing loss, and recurrent encephalopathy. She reported severe migraines about four times a year associated with marked photosensitivity, nausea, and vomiting (Table 1). She presented with stroke-like episodes and recurrent seizures at age 44 years, and was diagnosed with MELAS after the diagnosis was established in the proband and screening of family members was undertaken. At the age of 50 years, she showed features of cognitive decline with memory and speech deficits, and perseverative tendencies in speech. Her neurological examination was significant for a short attention span, impairments in immediate recall, diminished hearing acuity. Full range and normal extraocular movements, and normal fundoscopic examination were documented. Cranial nerve examination showed flattening of the right nasolabial fold, pronator drift in the right upper limb, spasticity in all four extremities, symmetrical deep tendon reflexes, and extensor plantar responses bilaterally. She continues to have a wide based apraxic gait. Her MRI studies showed progressive ex-vacuo dilatation of the ventricles, and generalized cerebral atrophy. Extensive punctate T2 signal abnormalities in the periventricular white matter, cerebral and cerebellar hemispheres and the temporal poles were also noted. She currently resides in a nursing home and is being treated with arginine, Coenzyme Q10, levocarnitine, thiamine, oral gliclazide for her diabetes, and multivitamins.

\section{I-2}

The proband's maternal grandmother was diagnosed with early onset of Alzheimer's disease at age 54 and was receiving supportive care at the time of the proband's diagnosis of MELAS. Her medical history included impaired hearing, angina, a myocardial infarction at 49 years, progressive memory difficulties, an appearance of premature ageing, asthma, and numerous allergies (Table 1). She was documented to have a complete left bundle branch block, left atrial hypertrophy and poor pedal pulses. On psychiatric assessment she was found to have severe echolalia and palilalia. She tended to perseverate in her responses. Her previous family history was unavailable as she was an adopted child. She died at the age of 65 from pneumonia. An autopsy was performed, the details of which are described in the pathology section.

\section{II-2}

The proband's elder maternal aunt has a history of headaches, diabetes mellitus, hearing loss and cardiac arrest requiring resuscitation (Table 1). When she was first seen, it was noted that she was of short stature but had no concerns with her exercise intolerance. She has suffered recurrent strokes. There appears to be premature aging. She has not been followed routinely by the clinic. 


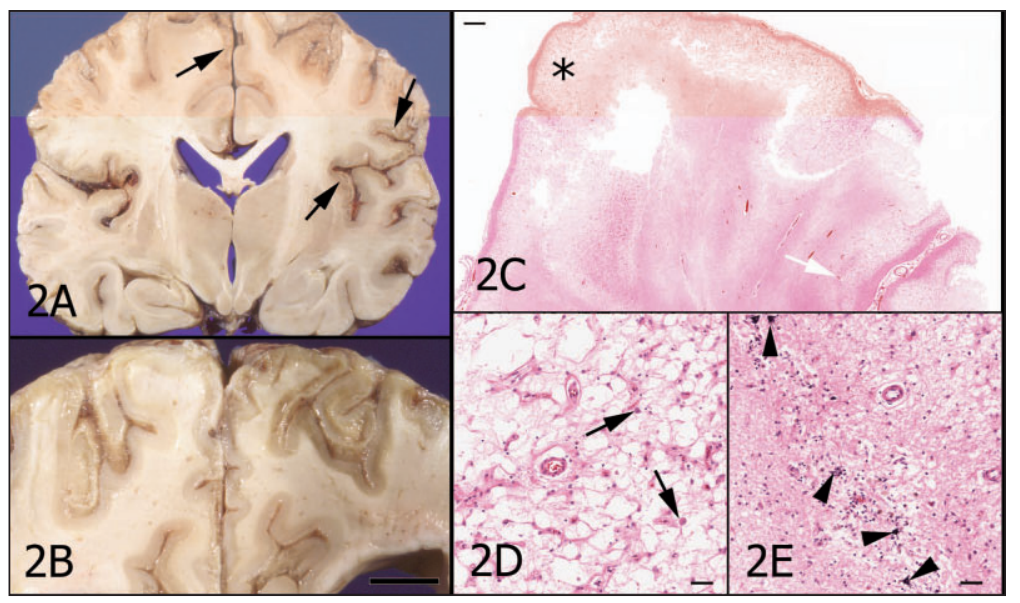

Figure 2: Brain: Gross and Microscopic Findings (Case III-1): Left hand panels (2A - coronal slice of the cerebral hemispheres at the level of the mammillary bodies; $2 B$ - coronal slice through the caudal parietal lobe at the level of the trigones): The brain is characterized by extensive but patchy softening, discoloration and laminar cortical necrosis (black arrows). These changes are most prominent in the dorsal half of the cerebral hemispheres and in the occipital region. Top right-2C (whole mount preparation, hematoxylin and eosin): There is marked rarefaction of the cortex (laminar 'necrosis') and a tendency for artifactual tearing to occur between the white and gray matter. The asterisk and white arrow indicate the site of sampling of the photomicrographs on the bottom left and right respectively. Note the 'pseudolaminar' pattern indicated by the white arrow. Bottom center (2D) and right (2E) (photomicrograph, hematoxylin and eosin, scale bar $50 \mu \mathrm{m}$ ); The most severely affected areas (left) are characterized by loosely arranged glial strands and scattered surviving neurons (black arrows). In other areas (right) the glial tissue is better preserved, there are mineralized neurons with a pseudolaminar distribution (black arrowheads) and there is a light lymphocytic infiltrate.

\section{II-1}

The proband's maternal uncle is an asymptomatic individual by report. He has not been evaluated formally in the neurometabolic clinic, and he has not been screened for mutation load in tissues or bodily fluids.

\section{II-6}

The proband's other maternal aunt remains clinically well, and reports no symptoms of hearing loss, diabetes, and stroke like episodes pertaining to MELAS. She too has not been formally evaluated in the neurometabolic clinic.

\section{III-3}

The proband's cousin, presently 20-years-old, was diagnosed with MELAS at four years-of-age when she presented with severe migraines which were experienced almost daily (Table 1). The migraines caused nausea and intense photophobia. These migraines were so intense in their nature that she had to miss school for a significant period. She has not had any major hospitalizations, seizures, or stroke like episodes. She is being treated with topiramate, calcium carbonate, vitamin D, vitamin C, Coenzyme Q10, vitamin B2, riboflavin, creatine monohydrate, alpha lipoic acid, and arginine. Her headaches are now mild and she has had only rare emergency room visits for breakthrough symptoms.

\section{III-4}

The younger cousin, now 17-years-old, has had a history of hand tremors, wrist injuries, fractures, significant depression and occasional headaches (Table 1). She is currently taking birth control pills, ciprazolam, reactine, and Coenzyme Q10.

\section{Molecular Studies}

Molecular studies were performed on blood lymphocytes in most of the individuals and in various other tissues from the autopsies of III-1 and I-2, such as cultured skin fibroblasts (Table 1). A 245 bp amplicon in MTTL1 was amplified using the polymerase chain reaction (PCR). The MTTL1 A3243G mutation introduces a cut site for restriction enzyme ApaI and the relative amounts of amplicon containing MTTL1 A3243G and the reference sequence were determined by quantitative densitometry after polyacrylamide gel electrophoresis and ethidium bromide staining ${ }^{4}$ (Table 1 ). The results suggest a great variation in mutation loads when different tissues and body fluids are assayed even in the same individual carrying the MTTL1 A3243G mutation.

\section{Post Mortem Tissue Handling}

Autopsies were carried out in both III-1 and I-2. For I-2, the tissues were taken for sampling 23.5 hours after death, and in III1,15 hours after death. In both cases the brain was removed and fixed in $20 \%$ formalin. The brain sections were embedded in paraffin wax, cut at eight microns and stained with hematoxylin and eosin. Skeletal muscle was also sampled for freezing and electron microscopy from the right quadriceps in III-I and the right rectus femoris in I-2. Electron microscopy was also carried on a cortical sample from I-2. The tissue for electron microscopy was fixed in glutaraldehyde at $4^{\circ} \mathrm{C}$, post-fixed in osmium tetroxide, embedded in Epon, and stained with uranyl acetate and lead citrate.

Frozen sections from the skeletal muscle were prepared using standard protocols. Specifically, the sections were stained with hematoxylin, phloxin and saffron and with Gomori modified trichrome; they were also treated with the following histochemical reactions: Acid phosphatase, ATPase $(\mathrm{pH} 4.2$, 9.6), cytochrome $\mathrm{C}$ oxidase (COX), Nicotinamide adenine dinucleotide (NADH), Periodic acid Schiff stain (PAS) and succinate dehdyrogenase (SDH).

\section{Post Mortem findings III-1}

The autopsy showed multifocal areas of cortical (predominantly occipital), cerebellar, and spinal cord necrosis with subependymal nodular heterotopias, temporal lobe tertial gyral dysmorphism and regional loss of myelinated fibres from the sural nerve. (Figure 2). 
Figure 3: Skeletal muscle, microscopic findings: Left panel, top$3 A$ (photomicrograph, hematoxylin and eosin, scale bar $50 \mu \mathrm{m}$ ): This high power view of the muscle illustrates the poor preservation of the tissue owing to post-mortem autolysis. However, many scattered skeletal muscle fibres throughout the sample show various degrees of 'foamy' or 'bland' degeneration (arrowheads). The skeletal muscle was taken from the right quadriceps in this case. Left panel, bottom-3B (photomicrograph, Gomori's modified trichrome, scale bar $50 \mu \mathrm{m}$ ): Although classic 'ragged' red fibres are not seen this staining method reveals small red inclusions (bottom arrowhead) and prominent red labelling of the enlarged mitochondria (upper arrowhead). Note that the indicated cells are the same as those illustrated in the top left panel. Central panel, top-3C (hematoxylin and eosin, scale bar $50 \mu \mathrm{m}$ ), right panel, top-3E (Gomori's modified trichrome, scale bar $50 \mu \mathrm{m}$ ), central panel, bottom-3D (succinic dehydrogenase histochemistry, scale bar $50 \mu \mathrm{m})$, right panel, bottom-3F (cytochrome $C$ oxidase, scale bar $50 \mu \mathrm{m}$ ). These photomicrographs are from serial sections that illustrate the various staining and histochemical characteristics of specific muscle fibres, identified by the arrowheads. The abnormal fibres are pale with hematoxylin and eosin, contain faint red granules with Gomori modified trichrome, have enhanced succinic dehydrogenase activity and exhibit reduced, normal or increased cytochrome C oxidase activity.

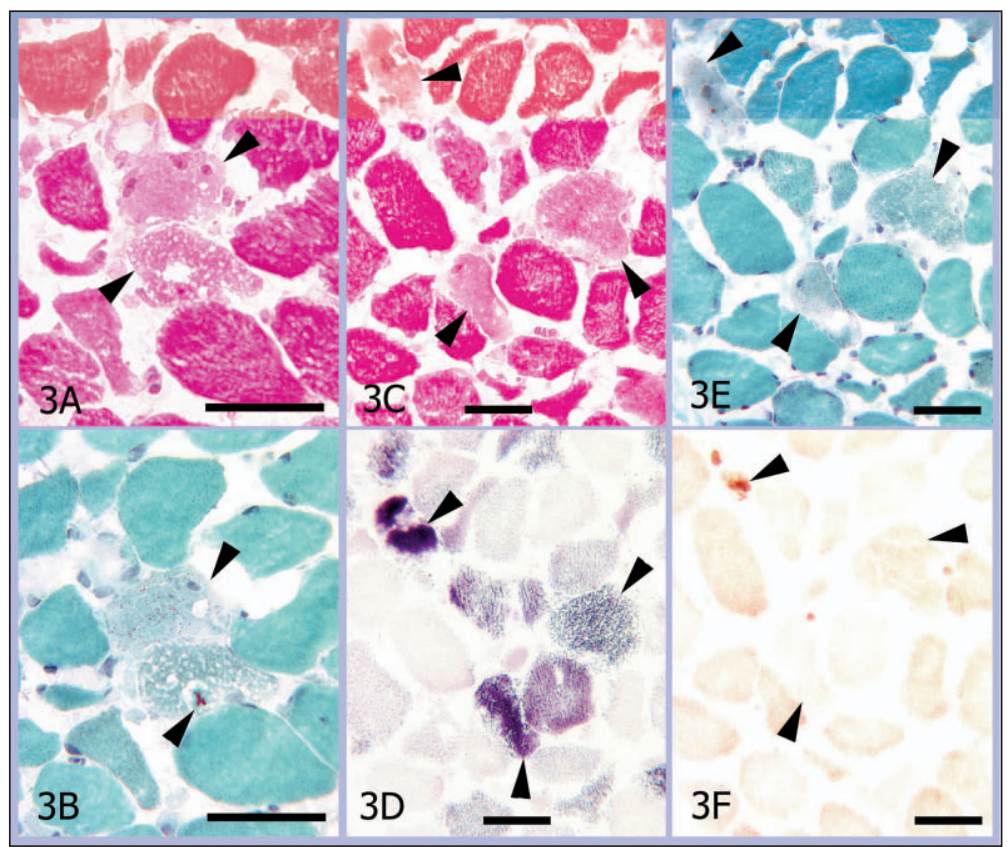

Muscle sampled at autopsy showed a 'necrotising myopathy' (i.e., scattered necrotic fibres) pattern with enhanced SDH and NADH activity and attenuated COX activity. In the proband's case, there were rare fibres with focally enhanced magenta staining in the sections stained with Gomori's modified trichrome method, but no true ragged red fibres (Figure 3).

\section{Post mortem findings I-2}

Autopsy findings confirmed the presence of Alzheimer's disease, mitochondrial myopathy, and findings related to respiratory complications from pneumonia as the terminal event.
The autopsy showed bilateral basal pneumonia, coronary atherosclerosis with occlusion of the left anterior descending branch of the coronary artery, evidence of an old left anterior myocardial infarct, congestive heart failure and right acute pyelonephritis.

Post mortem findings in the brain included widespread frontal, temporal, and parietal atrophy plus multiple small focal atheromatous plaques. In the meninges there was a moderate increase in subarachnoid fibrous connective tissue. In the cortex there was widespread spongiform change between layers one and two, extensive neuronal loss and marked astrogliosis.

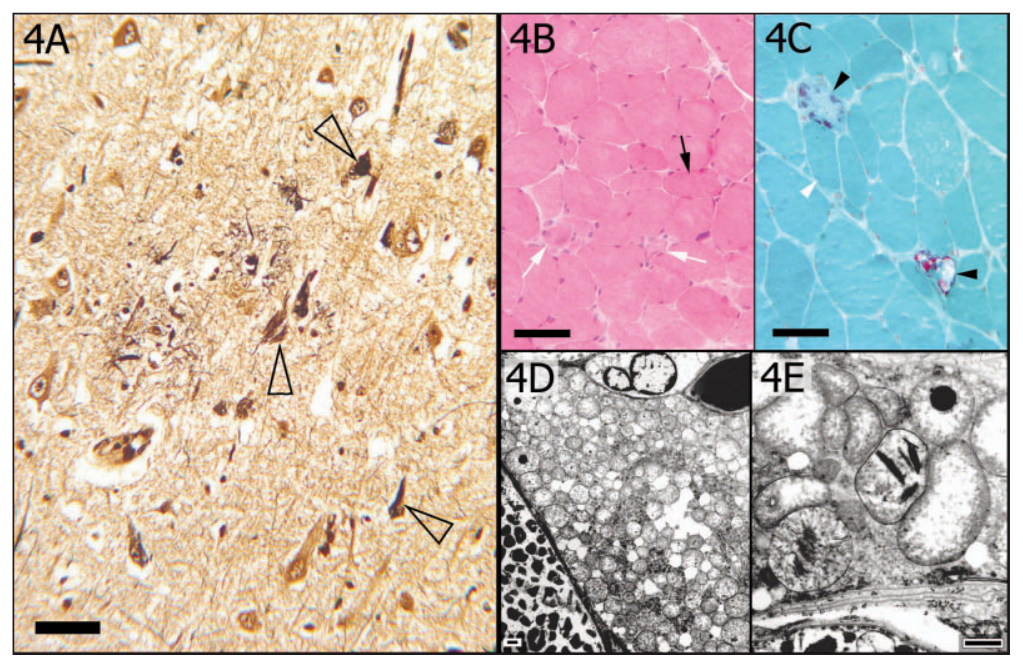

Figure 4: Microscopic and Ultrastructural Findings: Left hand panel-4A (photomicrograph, Bielschowsky method, scale bar 50 $\mu m):$ Numerous neurofibrillary tangles (open arrowheads) in sector CA1 of the hippocampus are shown. In addition, numerous neuropil threads are seen in the background and there are two illdefined neuritic plaques. Center panel, top-4B (photomicrograph, hematoxylin and eosin, scale bar $50 \mu \mathrm{m}$ ): There is mild variability in the calibre of the muscle fibres. Two fibres are degenerating (white arrows). The fibre indicated by the black arrow is slightly denser than many of the fibres and contains fine basophilic particulate material, which are the enlarged mitochondria. Right panel, top-4C (photomicrograph,

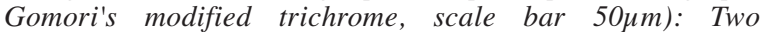
degenerating fibres exhibit red discoloration (black arrowheads), reminiscent of 'ragged red' fibres. Center panel, bottom-4D (electron micrograph, scale bar $1 \mu \mathrm{m})$ : The ultrastructure is poorly preserved because of post-mortem autolysis. Despite this, obvious large collections of subsarcolemmal mitochondria are visible. Right panel, bottom$4 E$ (electron micrograph, uranyl acetate/osmium tetroxide, scale bar $1 \mu \mathrm{m})$ : Details of the abnormal mitochondria are shown. Despite the poor preservation there is an impression in this and the previous frame that the cristae in some of the mitochondria are arranged radially. The endoplasmic reticulum is not demonstrated in relation to mitochondria. Electron dense material with various structural patterns is illustrated. 
Electron microscopic (EM) examination of the cortex revealed neurons with neurofibrillary tangles (NFT) (Figure 4); the mitochondria were poorly preserved but no inclusions were identified. There was widespread mild to moderate pallor in the white matter. There was severe loss of neurons from the hippocampus. The thalamus/basal ganglia showed mild perivascular atrophy. Examination of skeletal muscle showed mild myopathy associated with sparse ragged red fibres (Figure 4) which suggest a combinational deficiency in respiratory chain complexes I and $\mathrm{IV}^{5}$. The ultrastructure of the muscle was extremely poorly preserved and the assessment of the organelles unreliable; no paracrystalline inclusions seen and it was not possible otherwise to comment on the mitochondrial ultrastructure.

\section{MRI and MRS Findings III-2}

Initially restricted diffusion and high signal on Axial Fluid Attenuated Inversion Recovery (FLAIR) sequences were identified in the left occipital lobe (Figure 5B), but progressed over time to involve bilateral occipital lobes, and eventually multifocal changes involving bilateral hemispheres were documented (Figure 5C). Magnetic resonance spectroscopy showed the presence of an inverted doublet peak consistent with intracerebral accumulation of lactate in the region of the "stroke" (Figure 5D)

\section{Discussion}

This three-generation family demonstrates the clinical spectrum of MTTL1 A3243G ranging from asymptomatic individuals to patients with childhood and adult onset of MELAS. Current treatment is mainly directed towards symptom management and does not appear to alter the natural history of MELAS.

Mitochondria and their genome are localized in the cytoplasm of cells with most cell types having thousands of copies of the mitochondrial genome. At the tissue level and even the cellular level not all copies of the mitochondrial genome are necessarily of identical sequence (thus varying proportions of mutant and wild type mitochondria), resulting in heteroplasmy ${ }^{1}$. A consequence of heteroplasmy is that during cell division the percentage of mitochondrial DNA with a mutation may vary
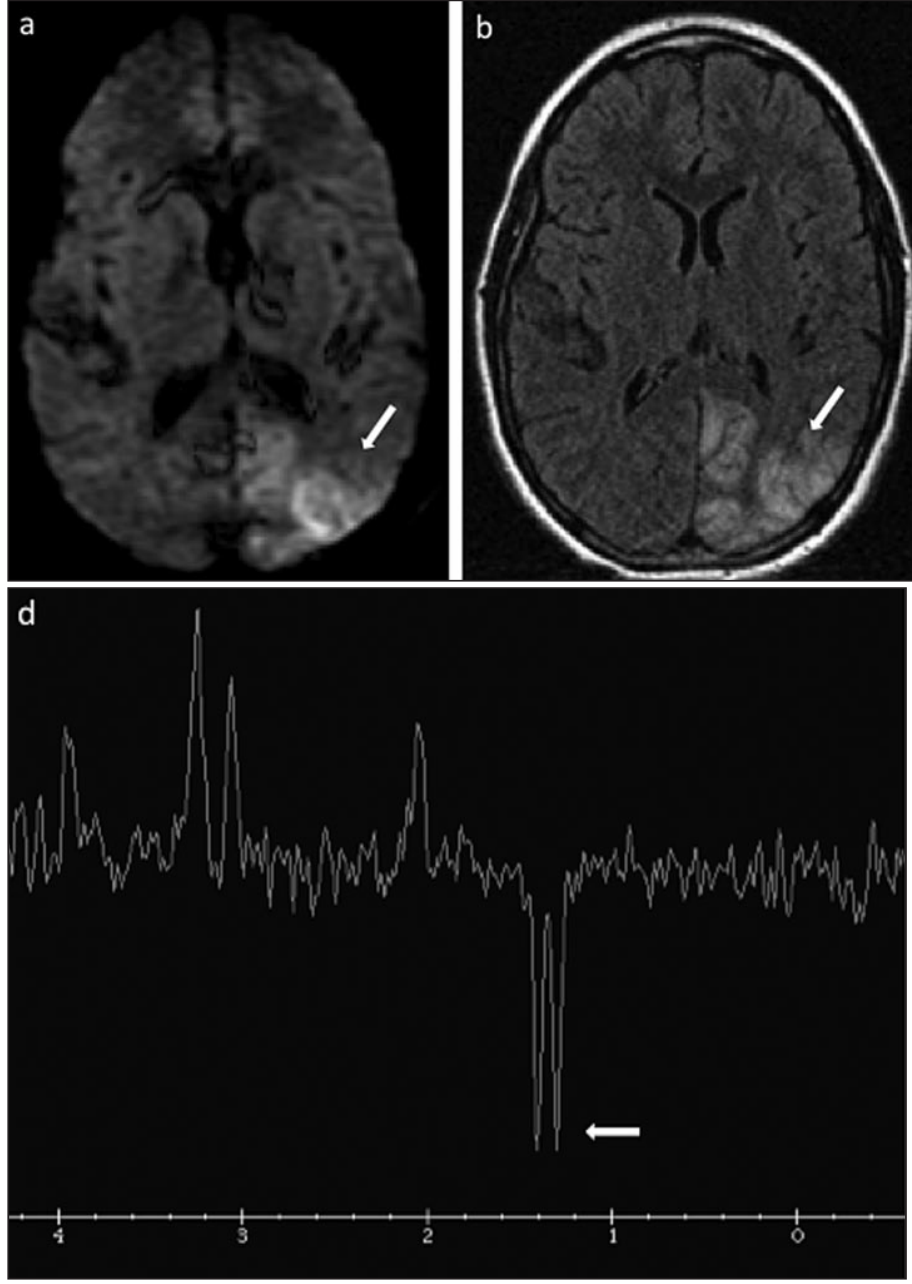

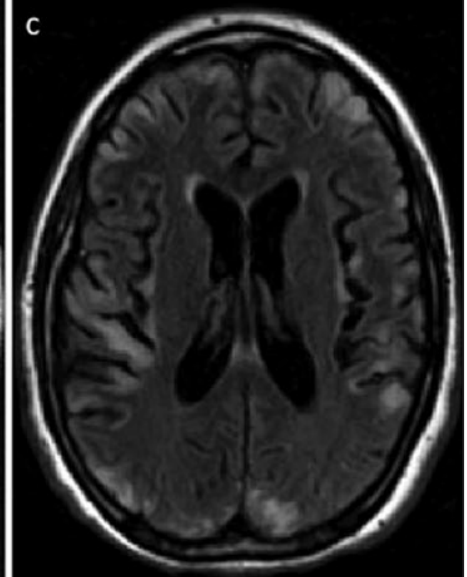

Figure 5:

a) Diffusion Weighted Imaging DWI and

b) Axial Fluid Attenuated Inversion Recovery (FLAIR) at presentation. Demonstrates restricted diffusion and hyperintensity in the left occipital lobe (white arrow) compatible with an infarct. c) Axial FLAIR sequence two years later demonstrates many more areas of brain parenchymal signal abnormalities and atrophy.

d) Single voxel MRS in the infarcted area shows inverted doublet of lactate (white arrow). 
between daughter cells when mitochondria are randomly segregated to each new cell. Tissues demonstrate the pathogenic effects of mutations when the load or proportion of mitochondrial DNA with a deleterious mutation exceeds critical threshold levels ${ }^{1}$. Threshold levels vary among tissues but typically tissues with higher energy requirements are most likely to be functionally compromised. In vitro studies with cybrids show that the MTTL $A 3243 G$ mutation impairs respiratory chain function as well as intramitochondrial protein synthesis when the mutant load exceeds the threshold level of about $85 \%{ }^{6}$. In the patients described in current family the maximum mutation burden was $84 \%$ in the 27 tissues analyzed (Table 1).

At conception, all mitochondria and mitochondrial DNA are contributed by the ovum making all attributes and diseases caused by mitochondrial DNA sequence changes to be inherited along the maternal lineage. The levels of MTTL1 A3243G decrease in blood cells with age presumably on the basis that cells with higher mutation burdens are less fit to divide ${ }^{7}$. However, there is evidence that mutational load generally increases with age in most other tissues ${ }^{3}$. The amount of mutant mitochondrial DNA also depends on the type of tissue it is present in. For example, it is usually higher in muscles in comparison to blood lymphocytes ${ }^{3}$.

In this multigenerational family, there appears to be a correlation between the age of onset and the percent of mutated DNA in blood leukocytes in affected individuals. Higher lactate levels particularly causing cerebral lactic acidosis are associated with more severe neurological impairment ${ }^{8}$. III- 1 and III-2 both carried a very significant burden of the neurological symptoms of MELAS that included frequent seizures and strokes and eventually succumbed to their illness following rapid neurological decline.

In a study by Chinnery et al, a positive correlation between the frequencies of common symptoms of MELAS, such as stroke, dementia, epilepsy and the mutant mtDNA load in muscle cells was noted. However, no such association between the frequency of clinical features and the amount of mutant mtDNA in blood was demonstrable ${ }^{9}$.

Another observation of significance is the difference in organ system tolerance to mutation loads. It has been observed that the load of mutant DNA in muscle is higher than in more rapidly dividing tissues (blood), which would explain the lack of correlation between the amount of mutant DNA in blood and clinical symptoms. However, there appears to be a discrepancy between in-vitro thresholds of mutant DNA loads for biochemical expression and in-vivo mutant loads resulting in clinical expression. Both I-2 and III-1 in the present family manifested with significant central nervous system (CNS) symptoms with mutant loads of around $67 \%$ in cortex (I-2) and $51-60 \%$ (Grey-white matter) in III-1.While III-1 had a higher mutation load in muscle (76\%), I-2 muscle appeared to have variable amounts of mutant mtDNA depending on the kind of muscle (skeletal or cardiac) and location of the muscle in the body (Table 1). This supports the observation that individuals with high mutation loads in the muscle are more likely to experience the more frequent symptoms of strokes and seizures.

A previous study attempting to determine the mutation thresholds of various tissues in patients afflicted with MELAS was performed ${ }^{3}$. Despite the direct association between the mutation loads in different tissues, and the clinical phenotype of
MELAS, it is possible that environmental and other genetic factors play a role in determining the final phenotypic expression of MELAS patients. For these reasons it is difficult to predict the prognosis for individual patients with mitochondrial DNA mutations.

There appears to be a regional susceptibility to strokes in MELAS. The occipital region appears to be more at risk for strokes and migraines. In the autopsy study of three patients with MELAS by Sparaco et al the most prominent cerebral immunocytochemical changes were found in the occipital regions of the patients, particularly in one with visual agnosia ${ }^{10}$. In the same study, it was found that mitochondrial abnormalities were found to be most prominent in the cerebral cortex affecting primarily the grey matter ${ }^{10}$. There was also reduced expression of COX-II and ATPase 8 in all three cases. The stroke-like episodes experienced by most patients correlate well with multiple often asymmetrical and multifocal infarct like lesions in the temporal, parietal and occipital regions ${ }^{11}$. Neuropathological characteristics of affected individuals include: multiple softening regions of different sizes and ages, (mostly contained in the cerebral cortex), basal ganglia calcification, spongiosis, cortical atrophy, multiple foci of infarction, mitochondrial angiopathy, and neuronal loss in both the cerebral and cerebellar cortex ${ }^{11-13}$. In current study, both the proband and his brother initially presented with occipital infarcts. The autopsy for the proband revealed predominantly foci of occipital necrosis, along with cerebellar and spinal cord lesions. In the case of his brother significant and prominent presentation during stroke-like episodes included visual symptoms with migrainous headaches. The role of mitochondrial angiopathy or vasculopathy deserves special attention as it is thought to be the basis for the stroke like episodes. This term was first used to describe an increase in the number of mitochondria in addition to being structurally abnormal in the cytoplasm of smooth muscle cells in the media as well as the endothelial cells of blood vessels in an autopsy study on two patients with MELAS. These changes were observed in pial arterioles and small arteries $250 \mu \mathrm{m}$ in size ${ }^{14,15}$.

A recently reported EM study of muscle has documented significant mitochondrial changes in endothelial cells of intramuscular capillaries. These changes include severe alterations in mitochondrial structure with fragmented and whorled cristae, and the presence of paracrystalline structures. The authors propose that the nature and distribution of these changes may further explain the lack of histochemical abnormalities in the muscle biopsies of young infants ${ }^{16}$. The role of microangiopathy or vasculopathy is further emphasized following the description of similar changes and vascular cell loss in cerebellar involvement and neurodegeneration in patients with mitochondrial DNA mutations ${ }^{17}$.

Muscle biopsies were performed during the autopsies of III-1 and I-2. Muscle biopsies have been shown to be the best method for confirming mitochondrial cytopathies 5 . The results would have been more accurate if they had been taken during life, but in III-1, due to previous molecular testing, it was already established that he had MELAS. It is important to point out that factors such as the nature of the tissue (fresh muscle vs, frozen tissue vs autopsy material), and careful attention to the use of standardized protocols (duration between time of death and sampling of tissue), and how pathological material is handled will ultimately affect the results of quantitative mitochondrial 
oxidative enzyme assays, as well as histochemical demonstration of enzyme deficiency. A detailed discussion of these factors is beyond the scope of this paper. There are established guidelines for the handling of such pathological material ${ }^{18}$. Ideally, quantitative assays of respiratory chain defects are best done on fresh muscle, however it should be remembered that a normal result of a quantitative assay in one tissue does not definitively exclude the diagnosis of mitochondrial disorders. The distinction between a primary defect in oxidative phosphorylation as opposed to a secondary defect from other neurodegenerative disorders also needs to be kept in mind. Current state of our knowledge suggests that molecular data from DNA studies can be more reliable in establishing a final diagnosis of mitochondrial disorders as was already evident in the present cases.

Mitochondria also appear to have a role in aging, (the maternal grandmother looked strikingly older in appearance than her chronological age would suggest). The mitochondria in her muscle cells were abnormally large; and it can be speculated that the mutation related defects in mitochondrial function could lead to both premature senescence and dementia ${ }^{19}$. The impairment of oxidative phosphorylation, accumulation of cerebral lactate, accumulation of reactive oxygen species, and the cumulative effects of multiple strokes over the patient's lifespan may be contributory to appearance of old age ${ }^{19-21}$.

Alzheimer disease has been described in MELAS patients, as has mtDNA encoded COX deficiency in Alzheimer patients ${ }^{22}$. Alzheimer type pathology has been found in a patient with MELAS $^{22}$. Neurofibrillary tangles were found in the para hippocampal gyrus and senile plaques were found throughout the brain. The patient in question had especially high amounts of mutated DNA in her brain, $89 \%$ was present in her frontal cortex (where the senile plaques were most frequently seen) ${ }^{22}$. In the current study, patient I-2, neurofibrillary tangles were found along with multiple plaques, however the levels of the mutated DNA in her anterior cortex was $67 \%$ (Table 1). Further research is needed to elucidate pathogenesis of both diseases and their relationship.

A number of treatments targeted at what are perceived to be the primary metabolic disruptions in mitochondrial diseases including MELAS have been proposed in either single case reports or small sized studies. Many of these treatments are based on exogenous supplements that are biologically logical as potential enhancers of mitochondrial energy production via the electron transport chain. In this family, patients II-4, III-1, III-2, III-3 and III-4 have been treated with many of these agents including vitamins $\mathrm{C}, \mathrm{E}$ and $\mathrm{K}$, Coenzyme Q10, succinic acid, creatine, arginine riboflavin, thiamin, carnitine, dichloroacetate, and lipoic acid. Pfeffer $e t a l$ in a recent analysis of treatments for mitochondrial diseases concluded that there was no clear evidence supporting any interventions ${ }^{23}$. This family also demonstrates that therapeutic efforts did not appear to attenuate the disease in the deceased individuals III- 1 and III-2, without a clear impact on disease progression in II- 4 . It is too early to make conclusions on III-3 and III-4. It is thought that stroke- like episodes are caused by the impairment of vasodilation in small cerebral arteries. These vessels are strong succinate dehydrogenase- reactive blood vessels (SSV's). Imaging studies using diffusion weighting and apparent diffusion coefficient
(ADC) maps favour the presence of vasogenic edema in acute phase of stroke like lesions encountered in MELAS. The SSV's and ragged red fibres in MELAS are COX positive. Hyperactive COX can bind regional nitric oxide (NO) and lead to lower NO concentrations that in turn leads to impaired flow mediated segmental vasodilation in the SSV's. In addition, the superoxide free radicals generated by the respiratory chain dysfunction can interact with $\mathrm{NO}$ to form neurotoxic peroxynitriles that cause neuronal cell death. The molecular pathogenicity has been summarised in an elegant review that would be of interest to the reader $^{24}$.

L-Arginine was found to be of benefit in the acute phase of strokes based on its function as a potent donor of $\mathrm{NO}^{25}$. Subsequently its benefits in improving outcomes in both acute and chronic phase of MELAS have been documented in several reports in patients that have demonstrated improvement in endothelial function by improving flow mediated vasodilatation $^{25-27}$. L-Arginine is an effective vasodilator and after administration in III-2's case, lower lactate levels were seen on magnetic resonance spectroscopy (MRS $)^{28}$. Hypocitrullinemia was documented in a study on MELAS patients in comparison to controls ${ }^{29}$. Citrulline has recently been shown to be a more effective vasodilator since it results in de novo production of arginine within the cell ${ }^{30}$. Idebenone, a powerful antioxidant has also been used in combination therapy with LArginine with a view to mopping up free radicals generated in respiratory chain defects in MELAS ${ }^{31}$.

Anticipatory guidance for managing patients with MELAS is essential. Avoiding the commonly used anticonvulsant valproic acid, or the anaesthetic agent propofol, and fasting, and along with prompt management of acute illness will help in restoring the patient to baseline.

The risk of a woman who is heteroplasmic for a mitochondrial DNA mutation to have a child with mitochondrial disease is difficult to determine. The percentage of mtDNA with a mutation in peripheral blood leukocytes may not predict the percentage of mitochondria with a mutation in a fertilized ovum because of the combined effects of heteroplasmy and a bottleneck in mitochondria population that occurs during oogenesis. There is evidence that the higher the levels of MTTL1 $A 3243 G$ in a woman's peripheral blood leukocytes the higher the risk of having an affected child ${ }^{3}$. In this study, II-4 has a higher mutation load in her blood leukocytes and her two sons were affected severely.

There is perhaps more promise in reproductive options being developed to reduce the risk of women with MTTL1 A3243G and other mitochondrial genome mutations from having a severely affected child. Poulton et al discussed the potential for using preimplantation genetic diagnosis or nuclear transfer to prevent the transmission of mitochondrial DNA diseases ${ }^{32}$. In support of pre-implantation diagnosis, the polar body mutational load has been identified to closely reflect the oocyte mutational load in a patient heteroplasmic with MTTL1 A3243G $G^{33}$. The technologies and outcomes of nuclear transfers are less well developed however Tachibana et al have recently described the replacement of mtDNA in human oocytes by spindle transfer and the subsequent fertilization and blastocyst development ${ }^{34}$. 


\section{Conclusion}

This family demonstrates the occurrence of heteroplasmy, phenotypic heterogeneity, and varied clinical burdens associated with MELAS. Maternal inheritance and concepts of heteroplasmy are further illustrated in this family. The variable presentation of MELAS makes diagnosing these disorders furthermore challenging. The risks of having a child with a mitochondrial mutation are significantly higher when the mother also carries that mutation burden in a higher percentage herself. MELAS remains an enigmatic disorder with many challenges in diagnosis and management. Genetic counselling and prenatal diagnosis options are complex and need to be addressed. Arginine given intravenously or orally on a long term basis, may provide some stabilization of clinical symptoms.

\section{ACKNOWLEDGEMENTS}

The authors thank the family for allowing us to share the information and to Dr. Ingrid Tein, Department of Neurology, Hospital for Sick Children, Toronto, for helpful discussions. The poster related to this family's details was presented at the United Mitochondrial Foundation meeting in 2010.

\section{REFERENCES}

1. Sproule DM, Kaufmann P. Mitochondrial encephalopathy, lactic acidosis, and strokelike episodes: basic concepts, clinical phenotype, and therapeutic management of MELAS syndrome. Ann N Y Acad Sci. 2008;1142:133-58.

2. Goto Y, Nonaka I, Horai S. A mutation in the tRNA(Leu)(UUR) gene associated with the MELAS subgroup of mitochondrial encephalomyopathies. Nature. 1990;348:651-3.

3. Finsterer J. Genetic, pathogenetic, and phenotypic implications of the mitochondrial A3243G tRNALeu(UUR) mutation. Acta Neurol Scand. 2007;116:1-14.

4. Pavlakis SG, Phillips PC, DiMauro S, De Vivo DC, Rowland LP. Mitochondrial myopathy, encephalopathy, lactic acidosis, and strokelike episodes: a distinctive clinical syndrome. Ann Neurol. 1984;16:481-8.

5. Sarnat HB, Marin-Garcia J. Pathology of mitochondrial encephalomyopathies. Can J Neurol Sci. 2005;32:152-66.

6. Chomyn A, Meola G, Bresolin N, Lai ST, Scarlato G, Attardi G. In vitro genetic transfer of protein synthesis and respiration defects to mitochondrial DNA-less cells with myopathy-patient mitochondria. Mol Cell Biol. 1991;11:2236-44.

7. Rahman S, Poulton J, Marchington D, Suomalainen A. Decrease of 3243 A-->G mtDNA mutation from blood in MELAS syndrome: a longitudinal study. Am J Hum Genet. 2001;68:238-40.

8. Kaufmann P, Shungu DC, Sano MC, et al. Cerebral lactic acidosis correlates with neurological impairment in MELAS. Neurology. 2004;62:1297-302

9. Chinnery PF, Howell N, Lightowlers RN, Turnbull DM. Molecular pathology of MELAS and MERRF. The relationship between mutation load and clinical phenotypes. Brain. 1997;120 (Pt 10): 1713-21.

10. Sparaco M, Simonati A, Cavallaro T, et al. MELAS: clinical phenotype and morphological brain abnormalities. Acta Neuropathol. 2003;106:202-12.

11. Betts J, Jaros E, Perry RH, et al. Molecular neuropathology of MELAS: level of heteroplasmy in individual neurones and evidence of extensive vascular involvement. Neuropathol Appl Neurobiol. 2006;32:359-73.

12. Yokoyama T, Hasegawa K, Obama R, Ishihara T, Yagishita S. MELAS with diffuse degeneration of the cerebral white matter: report of an autopsy case. Neuropathology. 2010;30:56-60.
13. Moran M, Moreno-Lastres D, Marin-Buera L, Arenas J, Martin MA, Ugalde C. Mitochondrial respiratory chain dysfunction: Implications in neurodegeneration. Free Radic Biol Med. 2012; 53:595-609.

14. Ohama E, Ohara S, Ikuta F, Tanaka K, Nishizawa M, Miyatake T. Mitochondrial angiopathy in cerebral blood vessels of mitochondrial encephalomyopathy. Acta Neuropathologica. 1987;74:226-33.

15. Mizukami K, Sasaki M, Suzuki T, et al. Central nervous system changes in mitochondrial encephalomyopathy: light and electron microscopic study. Acta neuropathologica. 1992;83:449-52.

16. Sarnat HB, Flores-Sarnat L, Casey R, Scott P, Khan A. Endothelial ultrastructural alterations of intramuscular capillaries in infantile mitochondrial cytopathies: "mitochondrial angiopathy". Neuropathology. 2012;32:617-27.

17. Lax NZ, Pienaar IS, Reeve AK, et al. Microangiopathy in the cerebellum of patients with mitochondrial DNA disease. Brain. 2012;135:1736-50.

18. Janssen AJ, Smeitink JA, van den Heuvel LP. Some practical aspects of providing a diagnostic service for respiratory chain defects. Ann Clin Biochem. 2003;40:3-8.

19. Finsterer J. Mitochondrial disorders, cognitive impairment and dementia. J Neurol Sci. 2009;283:143-8.

20. Finsterer J. Cognitive decline as a manifestation of mitochondrial disorders (mitochondrial dementia). J Neurol Sci. 2008;272: 20-33.

21. Finsterer J. Cognitive dysfunction in mitochondrial disorders. Acta Neurol Scand. 2012;126:1-11.

22. Kaido M, Fujimura H, Soga F, et al. Alzheimer-type pathology in a patient with mitochondrial myopathy, encephalopathy, lactic acidosis and stroke-like episodes (MELAS). Acta Neuropathol. 1996;92:312-18.

23. Pfeffer G, Majamaa K, Turnbull DM, Thorburn D, Chinnery PF. Treatment for mitochondrial disorders. Cochrane Database Syst Rev. 2012;4:CD004426.

24. Koga Y, Povalko N, Nishioka J, Katayama K, Yatsuga S, Matsuishi T. Molecular pathology of MELAS and L-arginine effects. Biochimica Biophys Acta. 2012;1820:608-14.

25. Koga Y, Ishibashi M, Ueki I, et al. Effects of L-arginine on the acute phase of strokes in three patients with MELAS. Neurology. 2002;58:827-8.

26. Koga Y, Akita Y, Nishioka J, et al. MELAS and L-arginine therapy. Mitochondrion. 2007;7:133-9.

27. Koga Y, Akita Y, Nishioka J, et al. L-arginine improves the symptoms of strokelike episodes in MELAS. Neurology. 2005; 64:710-12

28. Hirata K, Akita Y, Povalko N, et al. Effect of L-arginine on synaptosomal mitochondrial function. Brain Dev. 2008;30: 238-45.

29. Naini A, Kaufmann P, Shanske S, Engelstad K, De Vivo DC, Schon EA. Hypocitrullinemia in patients with MELAS: an insight into the "MELAS paradox". J Neurol Sci. 2005;229-230:187-93.

30. El-Hattab AW, Emrick LT, Craigen WJ, Scaglia F. Citrulline and arginine utility in treating nitric oxide deficiency in mitochondrial disorders. Mol Genet Metab. 2012;107:247-52.

31. Lekoubou A, Kouame-Assouan AE, Cho TH, Luaute J, Nighoghossian N, Derex L. Effect of long-term oral treatment with L-arginine and idebenone on the prevention of stroke-like episodes in an adult MELAS patient. Rev Neurol. 2011;167: $852-5$.

32. Poulton J, Chiaratti MR, Meirelles FV, Kennedy S, Wells D, Holt IJ. Transmission of mitochondrial DNA diseases and ways to prevent them. PLoS Genet. 2010;6 e1001066.

33. Poulton J, Kennedy S, Oakeshott P, Wells D. Preventing transmission of maternally inherited mitochondrial DNA diseases. BMJ. 2009;338:b94.

34. Tachibana M, Amato P, Sparman M, et al. Towards germline gene therapy of inherited mitochondrial diseases. Nature. 2013;493: 627-31. 